\title{
Adaptive Fuzzy Control for Uninterruptible Pow Supply with Three-Phase PWM Inverter
}

\author{
Feng-Yih $\mathrm{Hsu}^{1}$ and Li-Chen $\mathrm{Fu}^{1,2}$ \\ Dept. of Electrical Engineering ${ }^{1}$ \\ Dept. of Computer Science \& Information Engineering ${ }^{2}$ \\ National Taiwan University, Taipei, Taiwan, R.O.C.
}

\begin{abstract}
The major problems of uninterruptible power supply (UPS) with three pho $P W M$ inverter arise from unknown nonlinear loading to and phase couplings the inverters. In this paper, an adaptive fuzzy control is proposed to solve the problems. The control objective is to track the desired sine waveform regardless the versatile nonlinear loading, whose bound can be represented by a fuzzy rule-ba. The algorithm embedded in the proposed architecture can automatically update $t$ fuzzy control rules and, consequently, drive the tracking errors to a designated neig borhood of zero.
\end{abstract}

\section{Introduction}

To provide the reliable uninterruptible power supply (UPS) for the use of cor puters or factory automation systems has become more and more important. $T_{\text {i }}$ most common configuration of a UPS system consists of a dc source, a dc-ac inverte and an inductance-capacitance (L-C) filter. To establish a clean and reliable UP the waveform of the output voltage of the UPS system can only contain low tot harmonic distortion (THD) and can only have little variation even in the face varied nonlinear load. To achive the above characteristics, a well designed controll is needed in a UPS system to compensate for the different system characteristi resulting from the various (nonlinear) loads to and phase couplings of the inverter A straightforward approach to improve the performance is to use a feedforwal compensator to cancel the dynamics of the unknown load. Dead beat control hi been proposed for the simple linear system load [1]-[4]. For a complex or ill-defint load model, repetitive control can assure high performance in the steady-state col dition but results in poor transient response because of the long period to learn proper control law [5]. On the other hand, the conventional control in an analc UPS is to raise the control gain properly to robustify the stability as well as th performance for unknown load [6]. An alternative, however, exists which is to us an intellignet control methdology to suitably adjust the control gain in a responsir 
$y$ so as to compensate for the variation of characteristics due to load change. llowing this stream of thought, in this paper, an adaptive fuzzy control for a UPS item is proposed to achieve the aforementioned control goal.

\section{Problem Formulation}

A UPS system equipped with a three-phase PWM inverter is depicted in Figure which consists of an L-C filter at the inverter output, IGBT's to play as switching vices, a dc capacitor filter at the inverter input, and an unknown load at the L-C jer output. The variables $v_{r_{1}}, v_{r_{2}}$ and $v_{r_{3}}$, are the PWM modulation signals nich are the inputs to the PWM modulator in the figure, whereas the variables $1, v_{i 2}$ and $v_{i 3}$, are the inverter outputs satisfying the following equality:

$$
v_{i}=\frac{E_{d}}{2} v_{r}-v_{h},
$$

lere $v_{i}=\left[v_{i 1}, v_{i 2}, v_{i 3}\right]^{T}, v_{r}=\left[v_{r 1}, v_{r 2}, v_{r 3}\right]^{T}, v_{h}=\left[v_{h 1}, v_{h 2}, v_{h 3}\right]^{T}$ which denotes e voltage-drop vector at the on-state of IGBT, and $E_{d}$ is the dc voltage of the verter. From Fig. 1, the mathematic model of the UPS system can be formulated follows:

$$
\begin{aligned}
C_{p} \dot{v}_{c} & =i_{c}=i_{i}-i_{L} \\
L_{s} \dot{i}_{i} & =v_{i}-v_{c}-v_{N}
\end{aligned}
$$

here $v_{\mathrm{c}}=\left[v_{\mathrm{c} 1}, v_{\mathrm{c} 2}, v_{\mathrm{c}: 3}\right]^{\prime \prime}$ is the vector of output voltages, $v_{N}=\left[v_{n}, v_{n}, v_{n}\right]^{T}$ with $1=\frac{1}{3}\left(v_{i 1}+v_{i 2}+v_{i 3}\right)$ being the neutral voltage, $i_{c}=\left[i_{c 1}, i_{c 2}, i_{c 3}\right]^{T}$ is the vector of e capacitor currents, $i_{i}=\left[i_{i 1}, i_{i 2}, i_{i 3}\right]^{T}$ is the vector of the inverter currents, and $=\left[i_{L_{1}}, i_{L_{2}}, i_{l, 3}\right]^{T}$ is the vector of the load currents. By differentiating equation ), we can get the following equation:

$$
\ddot{v}_{c}=\frac{1}{C_{p}} \dot{i}_{c}=\frac{1}{C_{p}}\left(\dot{i}_{i}-\dot{i}_{L}\right)=\frac{1}{C_{p} L_{s}}\left(v_{i}-v_{c}-v_{N}\right)-\frac{1}{C_{p}} \dot{i}_{L}
$$

J substitute equation (1) into equation (4), we can then obtain the dynamic equaon of the CPS system as follows:

$$
\ddot{v}_{c}=\frac{1}{C_{p} L_{s}}\left(\frac{E_{d}}{2} v_{r}-v_{h}-v_{c}-v_{N}-L_{s} \dot{i}_{L}\right)
$$

The control goal is to let the output-voltage vector $v_{c}$ track the desired sineave vector $v_{c}^{*}=\left[v_{c_{1} 1}^{*}, v_{c 2}^{*}, v_{c 3}^{*}\right]^{T}=\left[v_{c 1}^{*}\left(2 \pi f_{\omega} t\right), v_{c 1}^{*}\left(\frac{2 \pi}{3}+2 \pi f_{\omega} t\right), v_{c 1}^{*}\left(\frac{4 \pi}{3}+2 \pi f_{\omega} t\right)\right]^{T}$, lhere $f_{\omega}$ is the frequency of the clesired sine-wave voltage. Let the tracking error zctor be denoted as $e_{v_{c}}=v_{c}^{*}-v_{c}=\left[e_{v_{c} 1}, e_{v_{c} 2}, e_{v_{c} 3}\right]^{T}$ and its time derivative as $j_{c}=\dot{v}_{c}^{*}-\dot{v}_{c}=\dot{v}_{c}^{*}-\frac{1}{C_{p}} i_{c}$. Then, the error dynamics of the system can be derived is follows:

$$
\ddot{e}_{v_{s}}=\ddot{v}_{c}^{*}+\frac{1}{C_{p} L_{s}}\left(v_{c}-\frac{E_{d}}{2} v_{r}+v_{N}+v_{h}+L_{s} \dot{i}_{L}\right)
$$


Define the desired capacitor current vector $i_{c}^{*}=C_{p} \dot{v}_{c}^{*}=\left[i_{c 1}^{*}, i_{c 2}^{*}, i_{c 3}^{*}\right]^{T}$, and def a new vector, $q$, as $q=e_{i_{c}}+\lambda e_{v_{c}}=\left[q_{1}, q_{2}, q_{3}\right]^{T}$, where $e_{i_{c}}=i_{c}^{*}-i_{c}$ is the curr tracking error and $\lambda$ is a positive constant. Then, a different error dynamic mor with respect to $q$ can be derived as follows:

$$
\begin{aligned}
\dot{q} & =i_{c}^{*}+\frac{\lambda}{C_{p}} e_{i_{c}}+\frac{1}{L_{s}}\left(v_{c}-\frac{E_{d}}{2} v_{r}+v_{N}+v_{h}\right)+\dot{i}_{L} \\
& =i_{c}^{*}+\frac{\lambda}{C_{p}} q-\left(\frac{\lambda^{2}}{C_{p}}-\frac{1}{L_{s}}\right) v_{c}-\frac{1}{L_{s}}\left(\frac{E_{d}}{2} v_{r}-v_{N}-v_{h}\right)+\dot{i}_{L}
\end{aligned}
$$

Generally, the load is passive and the load current $i_{L}$ will vary with resp to different loads. Hence, the $j$-th element of $i_{L}$ here is assumed, without loss generality, as a time-varying nonlinear function, $i_{L j}=g_{j}\left(v_{c j}, t\right)$. In turn, this le: to an explicit expression of the time derivative of the load current, $i_{L}$, as:

$$
\frac{d i_{L j}}{d t}=\frac{\partial g_{j}}{\partial v_{c j}} i_{c j}+\frac{\partial g_{j}}{\partial t}=\frac{1}{C_{p}} \frac{\partial g_{j}}{\partial v_{c j}} i_{c j}+\frac{\partial g_{j}}{\partial t}=g_{f_{j}} i_{c_{j}}+g_{t j}
$$

where $g_{f_{j}}=\frac{\partial g_{j}}{\partial v_{c j}}$ and $g_{t_{j}}=\frac{\partial g_{j}}{\partial t}$, respectively.

Assume that $\left|g_{t_{j}}\right| \leq \epsilon_{t},\left|v_{h j}\right| \leq \epsilon_{h}$, and $\left|g_{f_{j}}\right| \leq M_{v} \leq \bar{M}_{v}$, for $j=1,2,3$, wh $\bar{M}_{v} \gg \epsilon_{t}+\frac{1 \epsilon_{h}}{3 L_{\mathrm{s}}}, \epsilon_{h}$ and $\epsilon_{t}$ are some positive constants.

To remove the effect of $v_{n}$, let $v_{r 1}+v_{r 2}+v_{r 3}=0$ so that we can derive $v_{n}$ $\frac{v_{i_{1}}+v_{i_{2}}+v_{i_{3}}}{3}=\frac{-\left(v_{h_{1}}+v_{h_{2}}+v_{k_{3}}\right)}{3}$. Now, let the control law be designed as follows:

$$
\begin{aligned}
v_{r 1} & =\frac{2 L_{s}}{E_{d}}\left[i_{c 1}^{*}-\left(\frac{\lambda^{2}}{C_{p}}-\frac{1}{L_{s}}\right) v_{c 1}+f\left(v_{c 1}, q_{1}\right)\right] \\
& =\frac{2 L_{s}}{E_{d}}\left[i_{c 1}^{*}-K_{v} v_{c 1}+f\left(v_{c 1}, q_{1}\right)\right] \\
v_{r 2} & =\frac{2 L_{s}}{E_{d}}\left[i_{c 2}^{*}-K_{v} v_{c 2}+f\left(v_{c 2}, q_{2}\right)\right] \\
v_{r 3} & =-\left(v_{r 1}+v_{r 2}\right),
\end{aligned}
$$

where $K_{v}=\frac{\lambda^{2}}{C_{n}}-\frac{1}{L_{s}}$ is a constant gain, $f$ is the proposed adaptive fuzzy controll

\section{Adaptive Fuzzy Control}

Let $f\left(v_{c k}, q_{k}\right)$ be rewritten as follows:

$$
f\left(v_{c k}, q_{k}\right)=K_{q} q_{k}+\left(\left|i_{c k}\right|+\epsilon\right) \tau_{f}\left(v_{c k}, q_{k}\right),
$$

where $\epsilon$ is a sinall constant satisfying $\epsilon \geq \frac{\left(\epsilon_{t}+\frac{\tau_{c} h}{T_{t}}\right)}{M_{v}\left(v_{c k}\right)}$ for $k=1,2$ and $\tau_{f}\left(v_{c k}, q_{k}\right)$ the key adaptive furzy function. To simplify the notations, let $u=\left[u_{1}, u_{2}\right]^{T}$ wi $u_{1}=v_{c k}, u_{2}=q_{k}$, and $w=\tau_{f}\left(v_{c k}, q_{k}\right)$, where $v_{c k}$ and $q_{k}$ are the $k$-th elements $v_{c}$ and $q$, respectively. 
$s$ a general description of the fuzzy knowledge representation [10], a fuzzy rule consists of a collection of fuzzy If-then rules. Let $u$ denote as an input fuzzy or in the discourse universe $U_{u}$ and $L=\left\{L_{j}^{1}, \cdots, L_{j}^{\alpha_{j}}, \cdots, L_{j}^{l_{j}}\right\}$ as a family tzay sets associated with the membership functions $\mu_{L_{j}^{\alpha_{j}}}$ (see Fig. 2) correiding to the variable $u, j=1,2$, where $L_{j}^{\alpha_{j}}$ is obviously a fuzzy set in $L_{j}$. In tion, the centers of the family of fuzzy sets, $L_{j}$, are grouped into a set as $\bar{u}_{j}=$ $\left.\cdots, \bar{u}_{j}^{\alpha_{j}}, \cdots, \vec{u}_{j}^{j}\right\}$, where $\bar{u}_{j}^{\alpha_{j}}$ is a center satisfying $\bar{u}_{j}^{1}<\cdots<\bar{u}_{j}^{\alpha_{j}}<\cdots<\bar{u}_{j}^{l_{j}}$ s see Fig. 2). Let $L$ and $\bar{u}$ be both defined as product sets, $L=\prod_{j=1}^{2} L_{j}$ and of $\prod_{j=1}^{2} \bar{u}_{j}$, respectively, consisting of the families of fuzzy sets $L_{j}$ and the sets of ers $\bar{u}_{j}, j=1,2$. Then, an example of the $i$-th fuzzy rule is expressed as follows:

$$
R[i]: \text { If } u \text { is } L^{\alpha_{(i)}} \text {, then } w \text { is } Q^{\beta_{(i)}} \text {, }
$$

re $L^{\alpha_{(i)}} \in L, \alpha_{(i)}=\alpha_{1(i)} \times \alpha_{2(i)}$ is a product index associated with the $i$-th rule, $: \tau_{f}(u)$ is denoted as an output fuzzy variable. Let $Q=\left\{Q^{1}, \cdots, Q^{\beta}, \cdots, Q^{r}\right\}$ tenoted as a family of fuzzy sets associated with the membership functions corresponding to the output variable $w_{\text {\% }}$ with $Q^{\beta}$ being a fuzzy set in the ily $Q$ and $Q^{\beta_{(i)}} \in Q$ with $\beta_{(i)}$ being an integer index associated with the $i$ ule. Furthermore, let the sets of centers of the family of fuzzy sets, $Q$, be oted as $\bar{w}=\left\{\bar{w}^{1}, \cdots, \bar{w}^{\beta}, \cdots, \bar{w}^{r}\right\}$, where $\bar{w}^{\beta}$ is a center of the fuzzy set satisfying $<\cdots<\bar{w}^{\mathcal{\beta}}<\cdots<\bar{w}^{r}$. Generally speaking, $w$ can be expressed as follows: be ritten as:

$$
w=\tau_{f}=\sum_{i=1}^{\Upsilon} \bar{w}^{\beta_{(\mathrm{i})}} \xi_{i}(u)=\Theta^{T} \xi(u),
$$

re $\Theta=\left[\bar{w}^{\beta_{(2)}}, \cdots, \bar{w}^{\beta_{(r)}}\right]^{T}$ is regarded as a parameter vector and $\xi=\left[\xi_{1}, \cdots\right.$, $r$ as a regressive vector.

Though $w$ has been defined previously, there still exits the problem is how to armine the optimal $w$ in terms of appropriate choice of the parameters of $\Theta$. To en this difficulty, for a neighborhood of $u_{2}=0$ we define a new variable

$$
u_{2 \Delta}=q_{k_{\Delta}}= \begin{cases}u_{2}-\bar{a}, & \text { as } u_{2}<\bar{a} ; \\ u_{2}-\bar{b}, & \text { as } u_{2}>\bar{b} \\ 0, & \text { otherwise. }\end{cases}
$$

that $u_{2 \Delta}=\dot{u}_{2}$, as $u_{2 \Delta} \neq 0$ where $\bar{a}, \bar{b}$ are some constant satisfying $\bar{a} \leq \bar{u}_{2}^{\alpha_{2}^{0}}<$ $: \bar{u}_{2}^{\alpha_{2}^{0}+1} \leq \bar{b}$, where the index $\alpha_{2}^{0}$ indicates that two contiguous center points, $\bar{u}_{2}^{\alpha_{2}^{0}}$ $1 \bar{u}_{2}^{\alpha_{2}^{0}+1}$, are in the neighborhood of zero. Let $\phi$ represent the deadzone range, , $\phi=[\bar{a} \bar{b}]$. Based on this new variable definition, we can define the optimal ameter vector as follows:

$$
\Theta^{*}=\arg \min \left[\sup _{u \in U_{\Delta}} \Theta^{T} \xi(u) \operatorname{sgn}\left(u_{2}\right) \geq M_{v}\left(u_{1}\right)\right]
$$

ere $U_{\Delta}=U_{u} \backslash \phi$. After the optimal vector $\Theta_{k}^{*}$ is sought, we thus redesign $\left.\nu_{c k}, q_{k}\right)$ as

$$
f_{k}(u)=\left(\left|i_{c k}\right|+\epsilon\right)\left\{d_{k}(u) \tau_{f}(u)+\left[\begin{array}{c}
\left.1-d_{k}(u)\right] \\
191
\end{array}\right] \bar{M}_{v} \operatorname{sgn}\left(q_{k}\right)\right\}+K_{q} q_{k}, k=1,2
$$


where $u=\left[v_{c k}, q_{k}\right]^{T}, d=\left[d_{1}, d_{2}\right]^{T}$ and its $k$-th element $d_{k}$ is expressed as

$$
d_{k}(u)=\left\{\begin{array}{ll}
1, & \text { as } u \in U_{\Delta} \\
0, & \text { otherwise. }
\end{array},\right.
$$

Besides, the update laws are given as follows:

$$
\dot{\Theta}=r\left|i_{c k}\right| q_{k \Delta} \xi(u) \text { as } u \in U_{\Delta}
$$

for some $r>0$. The following theorem states the condition under which the abc mentioned adaptive fuzzy control law will yield satisfactory result.

Theorem 1 If the control law and the update law are given as in equations (9 (11), (14), (17) and (18), then the tracking errors will asymptotically converge $t$ neighborhood of zero.

\section{Simulation Results}

Consider a nonlinear load as a triac-connected resistor, whose resistor is giver $50 \Omega$ and with firing angles $\alpha$ as $36^{\circ}$ and $72^{\circ}$. Various coefficients of the UPS mo are $C_{p}=75 \mu F, L_{s}=0.5 m H, v_{h}=[2,2,2], E_{d}=400$, and the desired sine-w output voltage is given as $v_{c d}^{*}=\left[156 \sin (120 \pi t), 156 \sin \left(\frac{2 \pi}{3}+120 \pi t\right), 156 \sin \left(\frac{47}{3}\right.\right.$ $120 \pi t)]^{T}$. The triangular form and sup-min operator are selected as members functions and compositional operators, respectively. The total number of the $\mathrm{rl}$ of the fuzzy controller, $\Upsilon=l_{1} \times l_{2}=5 \times 5=25$, and the initial parameter vec of fuzzy-rule base, $\Theta$, is set to zero. The sampling time of control servo is given $50 \mu s$ in the outer loop, and the inner loop is to simulate the dynamic equation $w$ fixed step size $0.1 \mu \mathrm{sec}$. Fig. 3 shows the results of simulation results for firing an $36^{\circ}$. At the beginning, sinice initial parameter vector is set to zero, which appe to use only the PD controller to compensate for the uncertainties in the first per: of sine wave, the tracking error is quickly driven toward zero after the first peri

\section{Conclusions}

In the paper, we had proposed a novel fuzzy controller for a UPS system $w$ three-phase PWM inverter. It can update fuzzy control rules to compensate for: unknown nonlinear load and drive the tracking error of the output voltages to 1 designated neighborhood of "sero. The simulation results showed that the propop: fuzzy control scheme posseses a rapid converging feature. Besides, a dexterous । of fuzzy mathematics made the seeming complicated control be a scheme with : pealing computing efficiency.

\section{References}

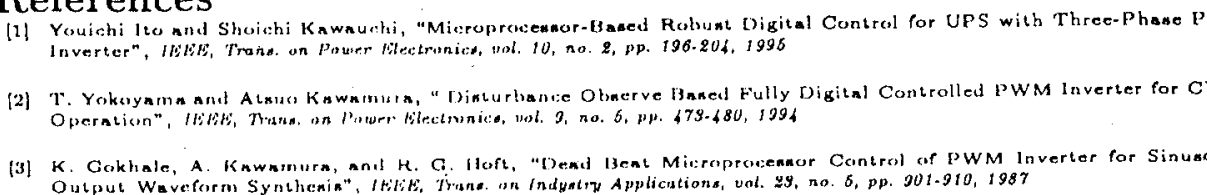


T. Kawatiate, T. Miymehita and Y. Yamamoto, "Dead Beat Control of Three Phase PWM Inverter", IEBE, Thans. on

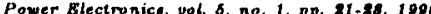

T. Haneyonhi nnd A. Kawamura, "Weveform Compenestion of PWM Inverter with Cyclic Fluctuating Lasda", IEEB Trans. on Indyetry Applications, wal. 24, no. 4, pp. 584-s89. 1980

M. J. Fyan and R. D. Lorens. "A lligh Performance Sino Weve Inverter Contraller with Capacitor Current Feedback and UACK-EMF Decoupling", IKEE PESC, pp. 607.619, 1996

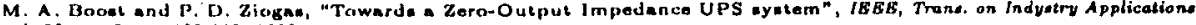
vol, 25, no. S, Pp. 408-418, 1989

L. D. Solvador, "A Multilevel Syatolle Appronch for Fussy Inference Hardware", IBEE Micro, pp. OS-71, October, 1990

T. Miki and T. Yamakawa "Fusay Inference on an Analog Funny Chip", IBBE Miero, pp. 8-18, Augual, 1995

L. X. Wang, "Stable Adaptive Fusey Control of Nonlinear Syetome", IBEE, Conf. an Decirion und Control, 1992 J-J E. Slatino and W. Li, Applied Nonlinear Control, Englowood Cliffe, NJ: Prantice Hall, pp. 278-284, 1991.

S. Sartry and M. Bodson, Adaptive Control, Stabitity, Convergence, and Robustnes, Prentico Hall, 1980.

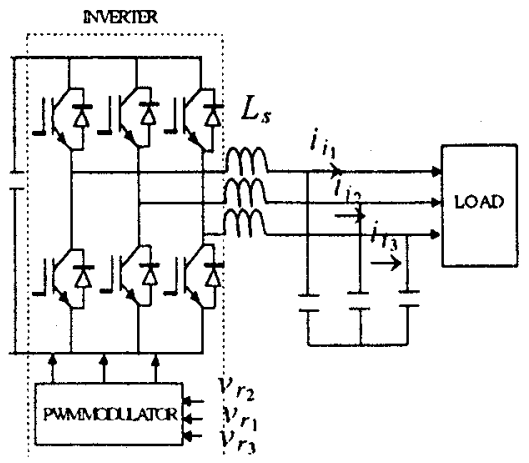

ure 1. U.JPS with a three-phase PWM inverter

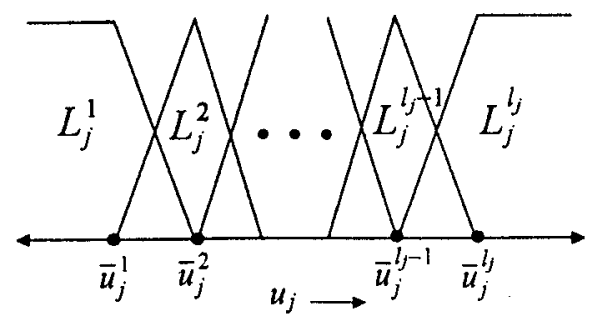

Figure 2. The environment of fuzzy variable $u_{j}$
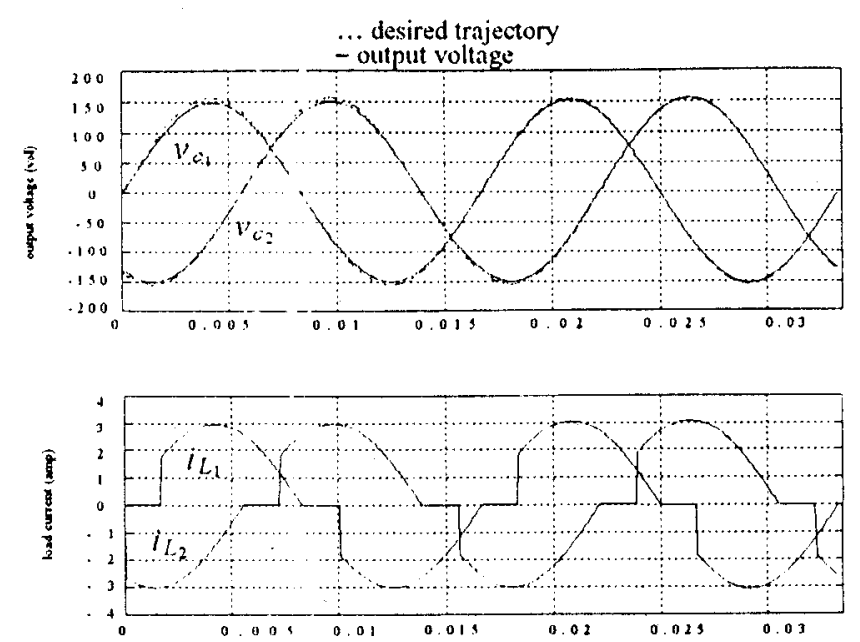

Figure 3. The simulation results 